\title{
A COUPLED MAGNETO-THERMO-ELASTIC PROBLEM IN A PERFECTLY CONDUCTING ELASTIC HALF-SPACE WITH THERMAL RELAXATION
}

\author{
S.K. ROY-CHOUDHURI \\ Department of Mathematics \\ Burdwan University \\ West Bengal, India \\ and \\ GARGI CHATTERJEE (ROY) \\ R.B.C. College, Nathati \\ West Benga 1 , India \\ (Received January 12, 1988 and in revised form August 14, 1989)
}

ABSTRACT. In the present paper we consider the magneto-thermo-elastic wave produced by a thermal shock in a perfectly conducting elastic half-space. Here the LordShulman theory of thermoelasticity [1] is used to account for the interaction between the elastic and thermal fields. The solution obtained in analytical form reduces to those of Kaliski and Nowacki [2] when the coupling between the temperature and strain fields and the relaxation time are neglected. The results also agree with those of Massalas and DaLamangas [3] In absence of the thermal relaxation time.

KEY WORDS AND PHRASES. Magneto-thermoelastic wave; Thermal relaxation time. 1980 AMS SUBJECT CLASSIFICATION CODE.

\section{INTRODUCTION.}

Kaliski and Nowacki [2] investigated the problem of magneto-thermo-elastic disturbances generated by a thermal shock in a perfectly conducting elastic half-space in contact with a vacuum. It was assumed that both in the medium and in the vacuum there acted an initial magnetic field parallel to the plane boundary of the half-space and there was no influence of coupling between temperature and strain fields.

Later, Massalas and Dalamangas [3] considered the same problem where the coupling between the temperature and strain fields was considered. Very recently Chatterjee and Roy Choudhuri [4] extended the problem [3] in generalized thermo-elasticity of Green and Lindsay taking into account the two relaxation times.

In the present paper we extend the problem [3] in generalized thermoelasticity by using the thermal relaxation time of Lord-Shulman theory [1]. The solutions for temperature distribution, deformation and perturbed magnetic field in the vacuum are obtained in analytical form in the first power of the magnetothermo-elastic coupling parameter $\varepsilon$ and relaxation parameter $\tau_{0}{ }^{\prime}$. In absence of $\varepsilon, \tau_{0}{ }^{\prime}$ the solutions agree with those in [2] and in absence of $\tau_{0}{ }^{\prime}$, the results agree with those in [3]. 
Surface stress for different times is calculated and graphically presented. It is believed that this particular problem has not been considered earlier.

2. PROBLEM FORUULATION.

We assume that a magneto-thermo-elastic wave is produced in an elastic halfspace $x_{1}>0$ due to the thermal shock $\theta(0, t)=\theta_{0} H(t)$ applied on $x_{1}=0$ where $\theta_{0}$ is a constant and $H(t)$ is the Heaviside function. We also assume that in both the media there is an initial magnetic field acting in the direction of $x_{3}$-axis. The simplifled equations of slowly noving bodies in electrodynamics after linearization are the following:

$$
\begin{aligned}
& \vec{\nabla} \times \vec{h}=\frac{4 \pi}{c} \vec{j} \\
& \vec{b} \times \vec{E}=-\frac{\mu O}{c} \frac{\partial \vec{h}}{\partial t} \\
& \vec{\nabla} \cdot \vec{h}=0, \vec{E}=-\frac{\mu_{0}}{c}\left(\overrightarrow{\vec{\mu}} \times \vec{H}_{0}\right)
\end{aligned}
$$

where $\vec{E}$ denotes the electric field, $\vec{h}$ is the perturbation of the magnetic field, $\overrightarrow{H_{0}}$ is the initial constant magnetic field, $\vec{f}$ is the current density vector, $\vec{u}$ denotes the displacement vector, $\mu_{0}$ is the magnetic permeability, $\sigma$ is the electric conductivity and $c$ is the velocity of light. The displacement equation of motion in thermoelasticity including the electromagnetic effect after linerization is,

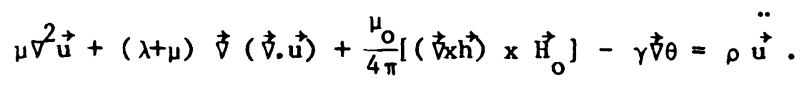

Also the modifled form of Fourler's law of heat conduction taking into account the thermal relaxation time $[1]$ is

$$
\rho_{v}\left(\dot{\theta}+\tau_{0} \ddot{\theta}\right)+\gamma T_{0}\left(\Delta+\tau_{0} \ddot{\Delta}\right)=K \theta, 11,(1=1,2,3)
$$

where $\lambda, \mu$ are the Lame' constants, $\gamma$ is equal to $(3 \lambda+4 \mu) \alpha_{T}, \alpha_{T}$ is the co-efficient of linear thermal expansion, $\theta$ is equal to $\mathrm{T}-\mathrm{T}_{0} ; \mathrm{T}_{0}, \mathrm{~T}$ are the reference and absolute temperature of the body respectively; $K$ is the co-efficient of heat conduction; $\rho$ is the mass density; $c_{v}$ is the specific heat at constant volume; $\tau_{0}$ is the relaxation time. The magneto-thermo-elastic wave propagated in the medium $x_{1}>0$ is assumed to depend on $x_{1}$ and time $t$.

For $\vec{H}_{0}=\left(0,0, \mathrm{H}_{3}\right)$ equations $(2.1)$ reduce to

$$
\vec{E}=\frac{\mu_{0} H_{3}}{c}\left(0, \dot{u}_{1}, 0\right), \vec{h}=-\frac{c}{\mu_{0}}\left(0,0, \frac{\partial E_{2}}{\partial x_{1}}\right), \vec{j}=\frac{c}{4 \pi}\left(0,-\frac{\partial h_{3}}{\partial x_{1}}, 0\right) .
$$

Equations (2.2) and (2.3) then lead to

$$
\left(\lambda+2 \mu+a_{0}^{2} \rho\right) \frac{\partial^{2} u_{1}}{\partial x_{1}^{2}}-\gamma \frac{\partial \theta}{\partial x_{1}}=\rho \ddot{u}_{1}
$$




$$
\rho c_{v}\left(\frac{\partial \theta}{\partial t}+\tau_{0} \frac{\partial^{2} \theta}{\partial t^{2}}\right)+\gamma T_{0}\left(\frac{\partial^{2} u_{1}}{\partial x_{1} \partial t}+\tau_{0} \frac{\partial^{3} u_{1}}{\partial x_{\partial t^{2}}}\right)=K \frac{\partial^{2} \theta}{\partial x_{1}^{2}}
$$

where $a_{0}=\sqrt{\frac{\mu_{0} \mathrm{H}_{3}^{2}}{4 \pi \rho}}$ is the Alfven wave velocity. For convenience, we shall use the notations $u_{1}=u, x_{1}=x$.

In vacuum the system of equations of electrodynamics are

$$
\begin{aligned}
& \left(\frac{\partial^{2}}{\partial x^{\prime 2}}-\frac{1}{c^{2}} \frac{\partial^{2}}{\partial t^{2}}\right) h_{3}^{0}=0 \\
& \left(\frac{\partial^{2}}{\partial x^{\prime 2}}-\frac{1}{c^{2}} \frac{\partial^{2}}{\partial t^{2}}\right) E_{2}^{o}=0
\end{aligned}
$$

where $\mathbf{x}^{\prime}=-\mathbf{x}$.

The components $\mathrm{T}_{11}$ and $\mathrm{T}_{11}^{\circ}$ of Maxwel1's stress tensor in elastic medium and in vacuum are

$$
T_{11}=-\frac{\mu o}{4 \pi} h_{3} H_{3} \text { and } T_{11}^{o}=-\frac{1}{4 \pi} h_{3}^{o} H_{3} \text {. }
$$

The normal mechanical and thermal stress is

$$
\sigma_{11}=(\lambda+2 \mu) \frac{\partial u}{\partial x}-\gamma \theta
$$

The boundary conditions to be satisifed are

$$
\begin{array}{ll}
\sigma_{11}+T_{11}-T_{11}^{\circ}=0, x=x^{\prime}=0 \\
E_{2}=E_{2}^{o} & , x=x^{\prime}=0 \\
\theta(o, t)=\theta_{0} H(t) . &
\end{array}
$$

3. SOLUTION OF THE PROBLEM.

To find the solution of the problem we now introduce the following notations and non-dimensional variables

$$
c_{1}^{2}=\frac{\lambda+2 \mu}{\rho}, c_{0}^{2}=a_{0}^{2}+c_{1}^{2}, \xi=\frac{c_{0} x}{k}, \tau=\frac{c_{0}^{2} t}{k}
$$




$$
\begin{aligned}
& U=\frac{C_{0}\left(\lambda+2 \mu+a_{0}^{2} \rho\right)}{k \gamma T_{0}} u, z=\frac{\theta}{T_{0}}, \varepsilon=\frac{\gamma^{2} T_{0}}{C_{\varepsilon}\left(\lambda+2 \mu+a_{0}^{2} \rho\right)}, k=\frac{K}{\rho C_{\nu}}, \\
& \tau_{0}^{\prime}=\tau_{0} \omega^{*}, \omega^{*}=\frac{\rho C_{\nu} c_{0}^{2}}{k}=\frac{C_{0}^{2}}{k}, C_{\varepsilon}=\rho C_{\nu} .
\end{aligned}
$$

The equations $(2.5)-(2.7)$ and boundary conditions $(2.8)-(2.10)$ become

$$
\begin{aligned}
& \frac{\partial^{2} U}{\partial \xi^{2}}-\frac{\partial Z}{\partial \xi}-\frac{\partial^{2} U}{\partial \tau^{2}}=0, \xi>0 \\
& \frac{\partial^{2} Z}{\partial \xi^{2}}-\frac{\partial Z}{\partial \xi}-\tau_{0}^{\prime} \frac{\partial^{2} Z}{\partial \tau^{2}}-\varepsilon \frac{\partial U}{\partial \xi \partial \tau}-\varepsilon \tau_{0}^{1} \frac{\partial^{3} U}{\partial \xi \partial \tau^{2}}=0, \xi>0 \\
& \frac{\partial h_{3}^{0}}{\partial \xi^{\prime 2}}-\beta^{2} \frac{\partial^{2} h_{3}^{0}}{\partial \tau^{2}}=0, \xi^{\prime}>0 \\
& \frac{\partial U}{\partial \xi}-z+\beta_{1} h_{3}^{0}=0, \quad \xi=\xi^{\prime}=0 \\
& \beta_{2} \frac{\partial^{2} U}{\partial \tau^{2}}-\frac{\partial h_{3}}{\partial \xi^{\prime}}=0, \xi=\xi^{\prime}=0, \\
& Z(0, \tau)=\frac{\theta^{\circ}}{T_{0}} H(\tau),
\end{aligned}
$$

where $\quad \beta_{1}=\frac{\mathrm{H}_{3}}{4 \pi \mathrm{T}_{0}}, \quad \beta_{2}=\frac{\mu_{0} \mathrm{H}_{3} \gamma \mathrm{T}}{\rho c^{2}}, \quad \beta=\frac{\mathrm{C}_{0}}{\mathrm{C}}, \xi^{\prime}=-\xi$.

Intial conditions in the new variables are

$$
U(\xi, 0)=0, z(\xi, 0)=0, \frac{\partial z(\xi, 0)}{\partial \xi}=0
$$

We now introduce a potential function $\phi$ defined by

$$
\mathrm{U}=\frac{\partial \phi}{\partial \xi} .
$$

Using (3.7) in (3.1) and then integrating we get

$$
z(\xi, \tau)=\left(\frac{\partial^{2}}{\partial \xi^{2}}-\frac{\partial^{2}}{\partial \tau^{2}}\right) \phi \text { in } \xi>0
$$


Using (3.7), the equation (3.2) leads to

$$
\frac{\partial^{2} z}{\partial \xi^{2}}-\frac{\partial z}{\partial \tau}-\tau_{0}^{\prime} \frac{\partial^{2} z}{\partial \tau^{2}}-\varepsilon \frac{\partial^{3} \phi}{\partial \xi^{2} \partial \tau}-\varepsilon \tau_{0}^{\prime} \frac{\partial^{4} \phi}{\partial \xi^{2} \partial \tau^{2}}=0
$$

In the Laplace transform domain the equations (3.8), (3.9) and (3.3) become

$$
\begin{aligned}
& \overline{\mathrm{Z}}(\xi, s)=\left(\frac{\partial^{2}}{\partial \xi^{2}}-s^{2}\right) \bar{\phi}, \xi>0 \\
& \left(\frac{\partial^{2}}{\partial \xi^{2}}-s-\tau_{0}^{\prime} s^{2}\right) \bar{z}=\varepsilon s\left(1+\tau_{0}^{\prime} s\right) \frac{\partial^{2-} \phi}{\partial \xi_{0}^{2}}, \xi>0 \\
& \overline{\mathrm{h}}_{3}{ }^{\circ}=c_{3} \mathrm{e}^{-\beta s \xi^{\prime}}, \quad \xi>0 .
\end{aligned}
$$

In Laplace transform domain, the boundary conditions (3.4) - (3.6) reduce to

$$
\begin{aligned}
& \frac{\partial^{2-} \phi}{\partial \xi^{2}}-\bar{z}+\beta_{1} \bar{h}_{3}^{0}=0, \xi=0 \\
& B_{2} s^{2} \frac{\partial \bar{\phi}}{\partial \xi}-\frac{\partial \bar{h}_{3}}{\partial \xi^{\prime}}=0, \xi=\xi^{\prime}=0 \\
& \overline{\mathrm{Z}}(0,8)=\frac{\theta_{0}}{T_{0}} \frac{1}{s} .
\end{aligned}
$$

Eliminating $\bar{z}$ from (3.10) and (3.11) we get

$$
\frac{\partial^{4-} \phi}{\partial \xi^{4}}-\left\{1+\varepsilon+s+(1+\varepsilon) \tau_{0}^{\prime} s\right\} s \frac{\partial^{2-} \phi}{\partial \xi^{2}}+s^{3}\left(1+\tau_{0}^{\prime} s\right) \bar{\phi}=0
$$

The equation (3.16) reduces to (31) in [4] on setting $\alpha^{\prime}=\alpha^{*^{\prime}}=\tau_{0}^{\prime}$. The general solution of (3.16) vanishing at $\xi=\infty$ is

$$
\bar{\phi}(\xi, s)=c_{1} e^{-\lambda_{1} \xi}+c_{2} e^{-\lambda_{2} \xi}, \quad \xi>0
$$

where $\lambda_{1}, \lambda_{2}$ are given by the roots of the equation

$$
\lambda^{4}-s\left\{1+\varepsilon+s+(1+\varepsilon) \tau_{0}^{1} s\right\} \lambda^{2}+s^{3}\left(1+\tau_{0}^{1} s\right)=0 .
$$

Hence

$$
\begin{aligned}
\lambda_{1,2} & =\left[\frac{s}{2}\left\{s+1+\varepsilon+\tau_{0}^{\prime} \varepsilon s+\tau_{0}^{\prime} s\right) \pm\left[\left(1+\varepsilon^{2} \tau_{0}^{\prime 2}+\tau_{0}^{\prime 2}+2 \varepsilon \tau_{0}^{\prime}+2 \varepsilon \tau_{0}^{\prime 2}-2 \tau_{0}^{\prime}\right) s^{2}\right.\right. \\
& \left.\left.\left.+2\left(\varepsilon-1+2 \varepsilon \tau_{0}^{\prime}+\tau_{0}^{\prime}+\varepsilon^{2} \tau_{0}^{\prime}\right) s+(1+\varepsilon)^{2}\right]^{1 / 2}\right\}\right]^{1 / 2} .
\end{aligned}
$$


The equation (3.19) agrees with that of (34) in [4] for $\alpha^{\prime}=\alpha^{*^{\prime}}=\tau_{0}^{\prime}$. For $\alpha^{\prime}=\alpha^{*^{\prime}}=0$, the equations (3.16), (3.19) are in agreement with that of (24) in [3]. Thus the equations $(3.1),(3.2),(3.16),(3.19)$ are more general in the sense that they incorporate the effect of thermal relaxation time of Lord-Shulman theory. From (3.10) using (3.17) we have

$$
\bar{z}(\xi, s)=C_{1}\left(\lambda_{1}^{2}-s^{2}\right) e^{-\lambda_{1} \xi}+C_{2}\left(\lambda_{2}^{2}-s^{2}\right) e^{-\lambda_{2} \xi}, \xi>0
$$

From the boundary conditions (3.13) - (3.15) taking into account (3.17) and (3.20) we obtain a linear algebraic system with respect to $C_{1}, C_{2}$ and $C_{3}$ as

$$
\begin{aligned}
& C_{1} s^{2}+C_{2} s^{2}+\beta_{1} c_{3}=0, \text { at } \xi=\xi^{\prime}=0 \\
& \beta_{2} s \lambda_{1} c_{1}+\beta_{2} s \lambda_{2} C_{2}-\beta C_{3}=0, \text { at } \xi=\xi^{\prime}=0 \\
& C_{1}\left(\lambda_{1}^{2}-s^{2}\right)+C_{2}\left(\lambda_{2}^{2}-s^{2}\right)=\frac{\theta_{0}}{T_{0} s} .
\end{aligned}
$$

The constants $C_{1}(1=1,2,3)$ being determined by $(3.21)-(3.23)$, the solutions for $\bar{\phi}, \bar{z}, \overline{\mathrm{U}}, \overline{\mathrm{h}}_{3}{ }^{\mathrm{o}}$ are given by

$$
\begin{aligned}
& \bar{\phi}\left(\xi, s, \varepsilon, \tau_{0}^{\prime}\right)=\frac{\theta_{0}}{T_{0}}\left[\frac{\left(s \beta+\beta_{1} \beta_{2} \lambda_{2}\right) e^{-\lambda_{1} \xi}-\left(s \beta+\beta_{1} \beta_{2} \lambda_{1}\right) e^{-\lambda_{2} \xi}}{s\left(\lambda_{1}-\lambda_{2}\right)\left(\beta_{1} \beta_{2} s^{2}+\beta\left(\lambda_{1}+\lambda_{2}\right) s+\beta_{1} \beta_{2} \lambda_{1} \lambda_{2}\right.}\right] \\
& \bar{z}\left(\xi, s, \varepsilon \tau_{0}^{\prime}\right)=\frac{\theta_{0}}{T_{0}}\left[\frac{\left.\lambda_{1}^{2}-s^{2}\right)\left(s \beta+\beta_{1} \beta_{2} \lambda_{2}\right) e^{-\lambda_{1} \xi}-\left(\lambda_{2}^{2}-s^{2}\right)\left(s \beta+\beta_{1} \beta_{2} \lambda_{1}\right) e^{-\lambda_{2} \xi}}{s\left(\lambda_{1}-\lambda_{2}\right)\left(\beta_{1} \beta_{2} s^{2}+\beta\left(\lambda_{1}+\lambda_{2}\right) s+\beta_{1} \beta_{2} \lambda_{1} \lambda_{2}\right.}\right] \\
& \bar{U}\left(\xi, s, \varepsilon, \tau_{0}^{\prime}\right)=\frac{\theta_{0}}{T_{0}}\left[\frac{\lambda_{2}\left(s \beta+\beta_{1} \beta_{2} \lambda_{1}\right) e^{-\lambda_{2} \xi}-\lambda_{1}\left(s \beta+\beta_{1} \beta_{2} \lambda_{2}\right) e^{-\lambda_{1} \xi}}{s\left(\lambda_{1}-\lambda_{2}\right)\left(\beta_{1} \beta_{2} s^{2}+\beta\left(\lambda_{1}+\lambda_{2}\right) s+\beta_{1} \beta_{2} \lambda_{1} \lambda_{2}\right.}\right], \xi>0 \\
& \bar{h}_{3}^{o}\left(\xi^{\prime} s, \varepsilon, \tau_{0}^{\prime}\right)=\frac{\theta_{0}}{T_{0}} \frac{s \beta_{2} e^{-\beta s \xi^{\prime}}}{\beta_{1} \beta_{2} s^{2}+\beta\left(\lambda_{1}+\lambda_{2}\right) s+\beta_{1} \beta_{2} \lambda_{1} \lambda_{2}}, \xi^{\prime}>0 .
\end{aligned}
$$

Since $\varepsilon, \tau_{0}^{\prime}<1$ for small thermo-elastic couplings, we expand the functions $\bar{z}, \bar{U}, \bar{h}_{3}^{\circ}$ into Maclaurian's series and retain the first two terms in the series expansion to obtain 


$$
\begin{aligned}
& \bar{z}\left(\xi, s, \varepsilon, \tau_{0}^{\prime}\right) \simeq \frac{\theta_{0}}{T_{0}}\left[\frac{e^{-\xi / s}}{s}+\varepsilon\left\{\frac{\beta e^{-\xi s}}{\left(\beta+\beta_{1} \beta_{2}\right)(s-1)^{2}}+\frac{\beta_{1} \beta_{2} e^{-\xi s}}{\left(\beta+\beta_{1} \beta_{2}\right) \sqrt{s}(s-1)^{2}}+\frac{e^{-\xi \sqrt{s}}}{s(s-1)^{2}}\right.\right. \\
& \left.+\frac{\beta_{1} \beta_{2}}{2\left(\beta+\beta_{1} \beta_{2}\right) s(s-1)}+\frac{\xi}{2} \frac{e^{-\xi \sqrt{s}}}{\sqrt{s}(s-1)}-\frac{e^{-\xi \sqrt{s}}}{2 s(\sqrt{s}-1)^{2}}-\frac{\beta e^{-\xi \sqrt{s}}}{2\left(\beta+\beta_{1} \beta_{2}\right) s(\sqrt{s}+1)^{2}}\right\} \\
& \left.+\tau_{0}{ }^{\prime}\left\{-\frac{\xi \sqrt{s} e^{-\xi \sqrt{s}}}{2}\right\}\right] \\
& +\frac{\xi \beta^{e^{-\xi \sqrt{s}}}}{\left(\beta+\beta_{1} \beta_{2}\right) s(s-1)^{2}}-\frac{\beta \mathrm{e}^{-\xi s}}{2\left(\beta+\beta_{1} \beta_{2}\right) s(s-1)^{2}}+\frac{\xi \beta \mathrm{e}^{-\xi s}}{2\left(\beta+\beta_{1} \beta_{2}\right)(s-1)^{2}}+\frac{\xi \beta_{1} \beta_{2} \mathrm{e}^{-\xi \sqrt{s}}}{2\left(\beta+\beta_{1} \beta_{2}\right) s(s-1)^{2}}+
\end{aligned}
$$$$
\frac{\xi \beta_{1} \beta_{2} e^{-\xi s}}{2\left(\beta+\beta_{1} \beta_{2}\right) \sqrt{s}(s-1)^{2}}-\frac{e^{-\xi \sqrt{s}}}{2 s \sqrt{s}(s-1)(\sqrt{s}-1)^{2}}+\frac{\beta e^{-\xi s}}{2\left(\beta+\beta_{1} \beta_{2}\right) s(s-1)(\sqrt{s}-1)^{2}}
$$$$
+\frac{\beta_{1} \beta_{2} e^{-\xi s}}{2\left(\beta+\beta_{1} \beta_{2}\right) s \sqrt{s}(s-1)(\sqrt{8}-1)^{2}}+\frac{\beta^{2} e^{-\xi s}}{2\left(\beta+\beta_{1} \beta_{2}\right)^{2} s(s-1)(\sqrt{s}+1)^{2}}-\frac{\beta e^{-\xi \sqrt{s}}}{2\left(\beta+\beta_{1} \beta_{2}\right) s \sqrt{s}(s-1)(\sqrt{8}+1)^{2}}
$$$$
\left.+\frac{\beta \beta_{1} \beta_{2} e^{-\xi s}}{2\left(\beta+\beta_{1} \beta_{2}\right)^{2} s \sqrt{s}(s-1)(\sqrt{s}+1)^{2}}\right\}+\tau_{0}^{\prime} \frac{e^{-\xi \sqrt{s}}}{2 \sqrt{s}(s-1)}-\frac{\xi e^{-\xi \sqrt{s}}}{2(s-1)}-\frac{\beta_{1} \beta_{2} e^{-\xi s}}{2\left(\beta+\beta_{1} \beta_{2}\right) \sqrt{s}(s-1)}
$$$$
\left.\left.-\frac{\beta e^{-\xi s}}{\left(\beta+\beta_{1} \beta_{2}\right)(s-1)^{2}}+\frac{\beta e^{-\xi s}}{\left(\beta+\beta_{1} \beta_{2}\right) \sqrt{s(s-1)^{2}}}\right\}\right]
$$

$$
\begin{aligned}
& \overline{\mathrm{h}}_{3}{ }^{\circ}\left(\xi^{\prime}, s, \varepsilon, \tau_{0}^{\prime}\right) \simeq \frac{\theta_{0}}{T_{0}}\left[\frac{\beta_{2} e^{-\beta s \xi^{\prime}}}{\left(\beta+\beta_{1} \beta_{2}\right) \sqrt{s}(\sqrt{s}+1)}-\varepsilon \frac{\beta \beta_{2} e^{-\beta s \xi^{\prime}}}{2\left(\beta+\beta_{1} \beta_{2}\right)^{2} \sqrt{s}(\sqrt{s}+1)^{3}}\right. \\
& \left.-\tau_{0}^{\prime} \frac{\beta_{2} \sqrt{s} e^{-\beta s \xi^{\prime}}}{2\left(\beta+\beta_{1} \beta_{2}\right)(\sqrt{s}+1)^{2}}\right]
\end{aligned}
$$

Taking inverse Laplace transform we obtain (Chatterjee (Roy) and Roy Choudhur1 [4], Hetnarsk1 [5], Oberhettiner and Badil [6]), 
574

S. K. ROY-CHOUDHURI AND G. CHATTERJEE

$$
\begin{aligned}
& Z\left(\xi, \tau, \varepsilon, \tau_{0}{ }^{\prime}\right) \simeq \frac{\theta_{0}}{T_{0}}\left[\operatorname{erfc}\left(\frac{\xi}{2 \sqrt{\tau}}\right)+\varepsilon \frac{\beta}{\epsilon_{\beta+\beta_{1} \beta_{2}}}(\tau-\xi) e^{(\tau-\xi)} H(\tau \xi)+\frac{\beta_{1} \beta_{2}}{\beta+\beta_{1} \beta_{2}}\left[\frac{\Gamma \xi}{\pi}\right.\right. \\
& +\left(\tau-\xi-\frac{1}{2}\right) e^{(\tau-\xi)} \operatorname{erf} \sqrt{\tau-\xi]} H(\tau-\xi)+\tau f_{1}(\xi, \tau)-\frac{\xi}{2} f_{2}(\xi, \tau)-f_{1}(\xi, \tau)+\operatorname{erfc}\left(\frac{\xi}{2 \sqrt{\tau}}\right) \\
& +\frac{\beta_{1} \beta_{2}}{2\left(\beta+\beta_{1} \beta_{2}\right)}\left[f_{1}(\xi, \tau)-\operatorname{erfc}\left(\frac{\xi}{2 \sqrt{\tau}}\right)\right]+\frac{\xi}{2} f_{2}(\xi, \tau)-\frac{1}{2} f_{3}^{l}(\xi, \tau) \\
& \left.\left.-\frac{\beta}{2\left(\beta+\beta_{1} \beta_{2}\right)} f_{3}^{\Pi}(\xi, \tau)\right\}-\tau_{0}^{\prime}\left\{\frac{\xi}{2} \frac{1}{4 \sqrt{\pi}}\left(\xi^{2}-2 \tau\right) \tau^{-5 / 2} e^{\frac{\xi^{2}}{4 \tau}}\right\}\right]
\end{aligned}
$$

$$
U\left(\xi, \tau, \varepsilon, \tau_{0}^{\prime}\right)=\frac{\theta_{0}}{T_{0}} f_{2}(\xi, \tau)-2 \sqrt{\frac{\tau}{\pi}} e^{-\left(\frac{\xi^{2}}{4 \tau}\right)}+\xi \operatorname{erfc}\left(\frac{\xi}{2 \sqrt{\tau}}\right)-\frac{\beta}{\beta+\beta_{1} \beta_{2}}\left(e^{\tau-\xi}\right) H(\tau \xi)
$$

$$
\begin{aligned}
& -\frac{\beta_{1} \beta_{2}}{\beta+\beta_{1} \beta_{2}}\left[e^{(\tau-\xi)} \text { erf } \sqrt{\tau-\xi}-2 \sqrt{\tau-\xi}\right] H(\tau-\xi)+\varepsilon\left[-\frac{\beta}{2\left(\beta+\beta_{1} \beta_{2}\right)}\left[f_{3}(\xi, \tau)-f_{2}(\xi, \tau)\right.\right. \\
& \left.+2 \sqrt{\pi} e^{-\left(\frac{\xi}{4 \tau}\right)}-\xi \operatorname{erfc}\left(\frac{\xi}{2 \sqrt{\tau}}\right)\right]+\frac{\xi \beta}{2\left(\beta+\beta_{1} \beta_{2}\right)}\left[\tau f_{1}(\xi, \tau)-\frac{\xi}{2} f_{2}(\xi, \tau)-f_{1}(\xi, \tau)+\operatorname{erfc}\left(\frac{\xi}{2 \sqrt{\tau}}\right)\right]
\end{aligned}
$$

$-\frac{\beta}{2\left(\beta+\beta_{1} \beta_{2}\right)}(\tau \xi-1) e^{(\tau-\xi)} H(\tau-\xi)+\frac{\xi \beta}{2\left(\beta+\beta_{1} \beta_{2}\right)}(\tau-\xi) e^{(\tau-\xi)} H(\tau-\xi)+\frac{\xi \beta_{1} \beta_{2}}{2\left(\beta+\beta_{1} \beta_{2}\right)}\left[\tau f_{1}(\xi, \tau)\right.$

$$
\left.-(\xi / 2) f_{2}(\xi, \tau)-f_{1}(\xi, \tau) \operatorname{terfc}\left(\frac{\xi}{2 \sqrt{\tau}}\right)\right]+\frac{\xi \beta_{1} \beta_{2}}{2\left(\beta+\beta_{1} \beta_{2}\right)}\left[\frac{\sqrt{\tau \xi}}{\pi}+\left(\tau-\xi-\frac{1}{2}\right) e^{(\tau-\xi)} \operatorname{erfc} \sqrt{\tau-\xi}\right] H(\tau-\xi)
$$

$+f_{4}(\xi, \tau)+4 \frac{7}{\pi} e^{-\left(\frac{\xi^{2}}{4 \tau}\right)}-\operatorname{erfc}\left(\frac{\xi}{2 \sqrt{\tau}}\right)(\tau-\xi)-(2 \tau-\xi) e^{(\tau \xi)} \operatorname{erfc}\left(\frac{\xi}{2 \sqrt{\tau}}-\sqrt{\tau}\right)$

$+\frac{\beta}{2\left(\beta+\beta_{1} \beta_{2}\right)}\left[\left(\tau \xi-\frac{3}{2}\right) \frac{\sqrt{\tau-3}}{\pi}-\frac{1}{2}(5 \tau-5 \xi-3) e^{(\tau-\xi)}-\frac{5}{2}\left(\tau-\xi-\frac{1}{2}\right) e^{(\tau-\xi)} \operatorname{erf} \sqrt{\tau \xi}\right.$

$\left.+\left(\frac{3}{2}(\tau-\xi)+(\tau-\xi)^{2}\right) \operatorname{erf} \sqrt{\tau-\xi}+\frac{3}{2}(\tau \xi)+(\tau-\xi)^{2}-1\right] H(\tau \xi)+\frac{\beta_{1} \beta_{2}}{2\left(\beta_{+} \beta_{1} \beta_{2}\right)}\left[\left(\tau-\xi-\frac{9}{4}\right) \quad \frac{\sqrt{\tau-\xi}}{\pi}\right.$

$-\frac{1}{2}(7 \tau-7 \xi-5) e^{(\tau-\xi)}-\frac{1}{2}\left(7 \tau-7 \xi-\frac{11}{2}\right) e^{(\tau-\xi)} \operatorname{erf} \sqrt{\tau \xi}+\left(\frac{5}{2}(\tau \xi)+(\tau \xi)^{2}\right) \operatorname{erf} \sqrt{\tau \xi}$ 


$$
\begin{aligned}
& \left.+\frac{5}{2}(\tau \xi)+(\tau-\xi)^{2}-2\right] H(\tau-\xi)+\frac{\beta^{2}}{2\left(\beta+\beta_{1} \beta_{2}\right)^{2}}\left[-\left(\tau \xi-\frac{3}{2}\right) \sqrt{\tau-\xi}-\frac{1}{2}(5 \tau-5 \xi-3) e^{(\tau-\xi)}\right. \\
& \left.+\frac{5}{2}\left(\tau \xi-\frac{1}{2}\right) e^{(\tau-\xi)} \operatorname{erf} \sqrt{\tau-\xi}-\left(\frac{3}{2}(\tau-\xi)+(\tau \xi)^{2}\right) \operatorname{erf} \sqrt{\tau-\xi}+\frac{3}{2}(\tau \xi)+(\tau \xi)^{2}-1\right] H(\tau \xi) \\
& -\frac{\beta}{2\left(\beta+\beta_{1} \beta_{2}\right)}\left[f_{5}(\xi, \tau)-4 \sqrt{\pi} e^{-\left(\frac{\xi^{2}}{4 \tau}\right)}-\operatorname{erfc}\left(\frac{\xi}{2 \sqrt{\tau}}\right)(2+\xi)-(2 \tau+\xi-2) e^{(\tau+\xi)} \operatorname{erfc}\left(\frac{\xi}{2 \sqrt{\tau}}+\sqrt{\tau}\right)\right] \\
& +\frac{\beta \beta_{1} \beta_{2}}{2\left(\beta+\beta_{1} \beta_{2}\right)^{2}}\left[\left(\tau-\frac{9}{4}\right) \sqrt{\frac{\gamma-\xi}{\pi}}+\frac{1}{2}(7 \tau-7 \xi-5) e^{(\tau-\xi)}-\frac{1}{2}\left(7 \tau-7 \xi-\frac{11}{2}\right) e^{(\tau-\xi)} \operatorname{erf} \sqrt{\tau-\xi}\right. \\
& \left.\left.+\left(\frac{5}{2}(\tau-\xi)+(\tau-\xi)^{2}\right) \operatorname{erf} \sqrt{\tau-\xi}-\frac{5}{2}(\tau-\xi)-(\tau-\xi)^{2}+2\right] H(\tau-\xi)\right\}++\tau_{0}^{\prime}\left\{\frac{1}{2} f_{2}(\xi, \tau)-\frac{\xi}{2} f_{1}(\xi, \tau)\right. \\
& -\frac{\beta_{1} \beta_{2}}{2\left(\beta+\beta_{1} \beta_{2}\right)} e^{(\tau-\xi)} \operatorname{erf} \sqrt{\tau-\xi} H(\tau-\xi)-\frac{\beta}{\beta+\beta_{1} \beta_{2}}(\tau-\xi) e^{(\tau-\xi)} H(\tau-\xi)+ \\
& \left.\left.\frac{\beta}{\beta\left(\beta+\beta_{1} \beta_{2}\right.}\left[\left((\tau-\xi)-\frac{1}{2}\right) e^{(\tau-\xi)} \operatorname{erf} \sqrt{\tau-\xi}+\sqrt{\frac{\tau-\xi}{\pi}}\right] H(\tau-\xi)\right\}\right] . \\
& h_{3}^{o}\left(\xi^{\prime}, \tau, \varepsilon, \tau_{0}^{\prime}\right) \simeq \frac{\theta_{0}}{T_{0}}\left[\frac{\beta_{2}}{\beta+\beta_{1} \beta_{2}} e^{\left(\tau-\beta \xi^{\prime}\right)} \operatorname{erf} c \sqrt{\tau-\beta \xi^{\prime}} H\left(\tau-\beta \xi^{\prime}\right)-\varepsilon\left\{-\frac{\beta \beta_{2}}{2\left(\beta+\beta_{1} \beta_{2}\right)^{2}} 2\left(\tau-\beta \xi^{\prime}\right) \sqrt{\frac{F-\beta \xi^{\prime}}{\pi}}\right.\right. \\
& \left.+\left[1-2\left(\tau-\beta \xi^{\prime}\right)^{2}\right] e^{\left(\tau-\beta \xi^{\prime}\right)} \operatorname{erf} c \sqrt{\tau-\beta \xi} H\left(\tau-\beta \xi^{\prime}\right)\right\}+\tau_{0}^{\prime}\left\{-\frac{\beta_{2}}{2\left(\beta+\beta_{1} \beta_{2}\right)}\left[\frac{1}{\sqrt{\pi\left(\tau-\beta \xi^{\prime}\right)}}\right.\right. \\
& \left.\left.-2\left(\tau-\beta \xi^{\prime}\right) e^{\left(\tau-\beta \xi^{\prime}\right)} \operatorname{erf} c \sqrt{\tau-\beta \xi^{\prime}}+2 \sqrt{\left.\frac{\tau-\beta \xi^{\prime}}{\pi}\right]} H\left(\tau-\beta \xi^{\prime}\right)\right\}\right]
\end{aligned}
$$

where the functions $f_{1}(\xi, \tau), i=1,2,3,4,5$ are given by

$$
f_{1}(\xi, \tau)=e^{\tau}{ }^{\tau}\left[e^{-\xi} \operatorname{erfc}\left(\frac{\xi}{2 \sqrt{\tau}}-\sqrt{\tau}\right)+e^{\xi} \operatorname{erfc}\left(\frac{\xi}{2 \sqrt{\tau}}+\sqrt{\tau}\right)\right]
$$




$$
\begin{aligned}
& f_{2}(\xi, \tau)=\frac{e^{\tau}}{2}\left[e^{-\xi} \operatorname{erfc}\left(\frac{\tau}{2 \sqrt{\tau}}-\sqrt{\tau}\right)-e^{\xi} \operatorname{erfc}\left(\frac{\xi}{2 \sqrt{\tau}}+\sqrt{\tau}\right)\right] \\
& f_{3}^{I}(\xi, \tau)=\operatorname{erfc}\left(-\frac{\xi}{2 \sqrt{\tau}}\right)+2 \sqrt{\frac{\tau}{\pi}} e^{-\left(\frac{\xi^{2}}{4 \tau}\right)}+(2 \tau-\xi-1) e^{(\tau-\xi)} \operatorname{erfc}\left(\frac{\xi}{2 \sqrt{\tau}}-\sqrt{\tau}\right) \\
& \mathrm{f}_{3}^{\mathrm{II}}(\xi, \tau)=\operatorname{erfc}\left(\frac{\xi}{2 \sqrt{\tau}}\right)-2 \sqrt{\frac{\pi}{\pi}} e^{-\left(\frac{\xi^{2}}{4 \tau}\right)}+(2 \tau+\xi-1) e^{(\pi+\xi)} \operatorname{erfc}\left(\frac{\xi}{2 \sqrt{\tau}}+\sqrt{\tau}\right) \\
& f_{4}(\xi, \tau)=\int_{0}^{\tau} e^{m}\left\{2 \sqrt{\frac{\tau-m}{\pi}} e^{-\frac{\xi^{2}}{4(\tau-m)}}+[2(\tau-m)-\xi] e^{(\tau-\xi-m)} \operatorname{erfc}\left(\frac{\xi}{2 \sqrt{\tau-m}}-\sqrt{\tau-m}\right)\right] d m \\
& f_{5}(\xi, \tau)=\int_{0}^{\tau} e^{m}\left\{2 \sqrt{\frac{\tau m}{\pi}} e^{-\frac{\xi^{2}}{4(\tau-m)}}-[2(\tau-m)+\xi] e^{(\tau+\xi-m)} \operatorname{erfc}\left(\frac{\xi}{2 \sqrt{\tau-m}}+\sqrt{\tau-m)} d m\right.\right.
\end{aligned}
$$

where erfx and erfcx denote the error function and complementary error function respectively.

4. NUMERICAL RESULT.

The surface stress is given by

$$
\begin{aligned}
-\frac{T_{11}^{o}}{4 \pi T_{0} \theta_{3}} \frac{\beta_{2}}{B\left(1+\beta_{3}\right)} & =e^{\tau}\left(1-\operatorname{erf} \sqrt{\tau}-\frac{\varepsilon}{2\left(1+\beta_{3}\right)}\left\{-2 \tau \frac{\sqrt{\tau}}{\pi}+\left(1-2 \tau^{2}\right) e^{\tau}(1-\operatorname{erf} \sqrt{\tau})\right\}\right. \\
& -\tau_{0}^{\prime}\left\{\frac{1}{2 \sqrt{\pi \tau}}-\tau e^{\tau}(1-\operatorname{erf} \sqrt{\tau})+\sqrt{\frac{\pi}{\pi}}\right\}
\end{aligned}
$$

where $\quad B_{3}=\frac{B_{1} \beta_{2}}{\beta}$.

If there is no coupling between the electromagnetic field and strain field, $H_{3}=0, B_{2}=0, \beta_{3} \rightarrow 0$ and $B 18$ finite so that $T_{11}^{0}=0$ on $\xi=a_{0}$. In presence of the electomagnetic field and strain field, the surface stress is given by

$$
-\frac{T_{11}^{o}}{\frac{\theta_{0} H_{3}}{4 \pi T_{0}} \frac{B_{2}}{B\left(1+\beta_{3}\right)}}=X\left(\tau, \varepsilon, \tau_{0}^{\prime}\right)
$$


where

$$
\begin{aligned}
& \mathrm{X}\left(\tau, \varepsilon, \tau_{0}^{\prime}\right)=e^{\tau}(1-\operatorname{erf} \bar{\tau})-\frac{\varepsilon}{2\left(1+\beta_{3}\right)}\left\{-2 \tau \sqrt{\frac{\tau}{\pi}}+\left(1-2 \tau^{2}\right) e^{\tau}(1-\operatorname{erf} \sqrt{\tau})\right\} \\
& -\tau_{0}^{1}\left\{\frac{1}{2 \sqrt{\tau \pi}}-\tau e^{\tau}(1-\operatorname{erf} \sqrt{\tau})+\sqrt{\tau}\right\}
\end{aligned}
$$

We can assume $B_{3} \ll 1$ since $c \gg 1$ and $a_{0}$ and $c_{0}$ are finite. We take $B_{3}=.05$. For numerical calculation we take the material of the half-space to be copper for which $\varepsilon=0.0168$. $I_{i}$ we assume that a representative value of the relaxation time $\tau_{0}$ is $10^{-11}$ (see [7]), then the non-dimensional thermal wave speed in copper should be approximately equal to 0.66 . [8]).

Then $\tau_{0}^{\prime} \simeq 2.3$ (For thermal properties and sound wave speed in copper, see ref.

Surface stress $X$ for various values of times $\tau$ are exhibited in the following table and also graphically represented.

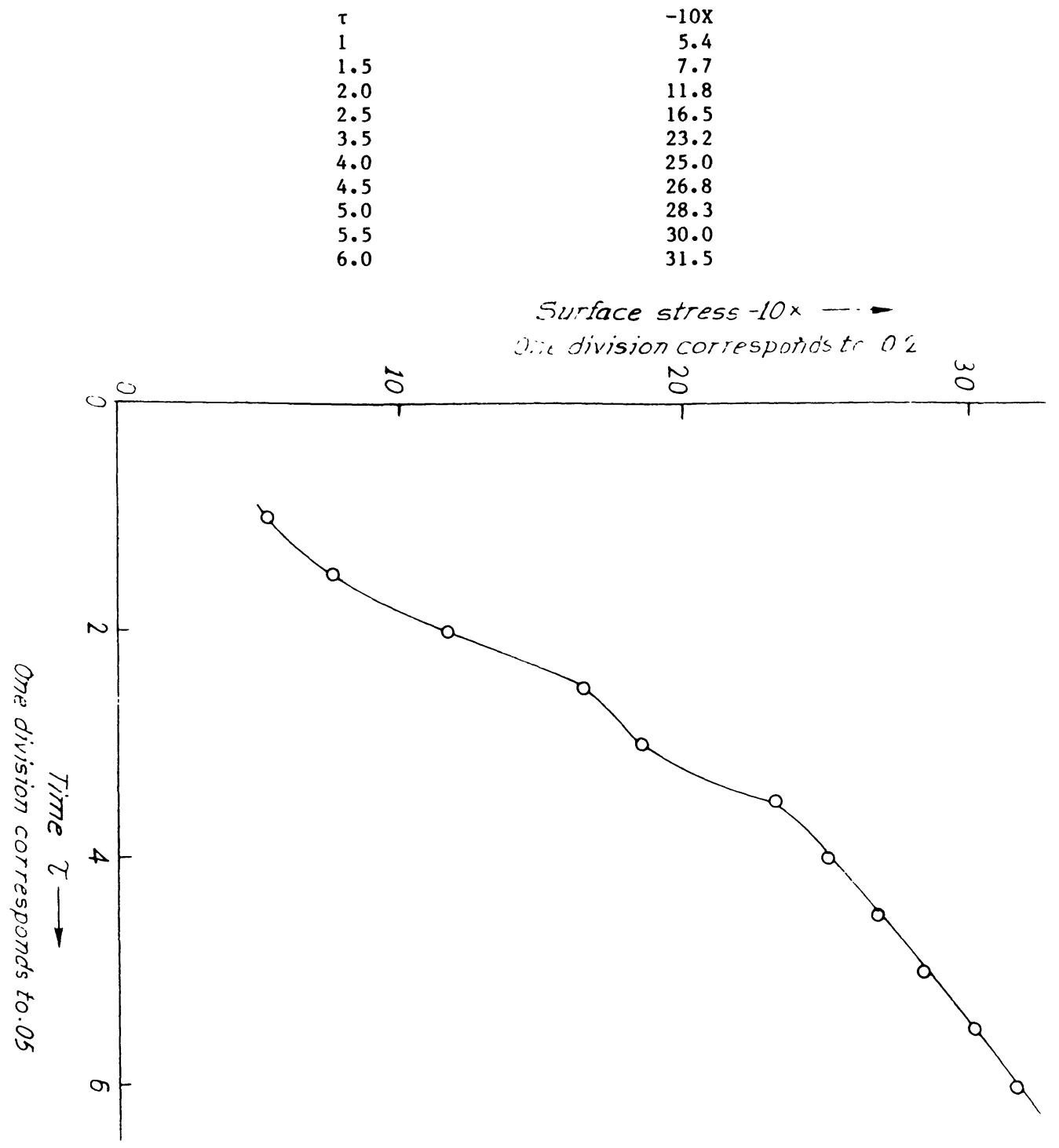




\section{REFERENCES}

1. LORD, H.W. and SHULMAN, Y., A Generalized Dynamical Theory of Thermoelasticity, J. Mech. Phys. Solids 15 (1967).

2. KALISKI, S. and NOWACKI, W., Excitation of Mechanical-electromagnetic Waves Induced by a Thermal Shock, Bull. Acad. Polon. Sci., Series Sci. Tech., 1, 10, (1962).

3. MASSALAS, C. and Dalamangas, A., Coupled Magneto-thermoelastic Problem in Elastic Half-space, Lett, Appl. Engg, Sc1. 21(2), (1983).

4. CHATTERJEe, G.(Roy), and ROY CHOUDHURI, S.R., Coupled Magneto-thermoelastic Problem in Elastic Half-space with Two Relaxation Times, Lett. Appli. Engg. Sci. 23(9), (1985).

5. HETNARSKI, R.B., Solution of the Coupled Problem of Thermoelasticity in the Form of Series of Functions, Arch. Mech. Stos, 4(16), (1964).

6. BADII, L. and OBERHETTINGER, F., Tables of Laplace Transforms, Springer-Verlag, 1973.

7. NAYFEH, A.H. and NEMAT-NASSER, S., Electromagento-thermoelastic Plane Waves in Solids with Thermal Relaxation, Jr. of Applied Mechanics 39(1) (1972).

8. AMERICAN INSTITUTE OF PHYSICS Handbook McGraw-Hi11, New York, 1957. 


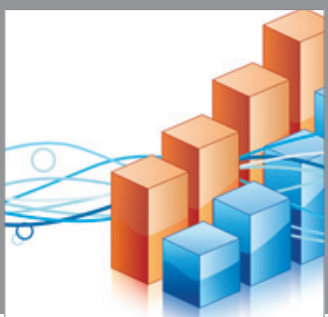

Advances in

Operations Research

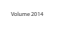

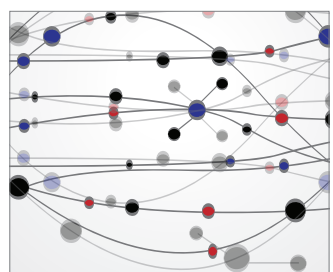

\section{The Scientific} World Journal
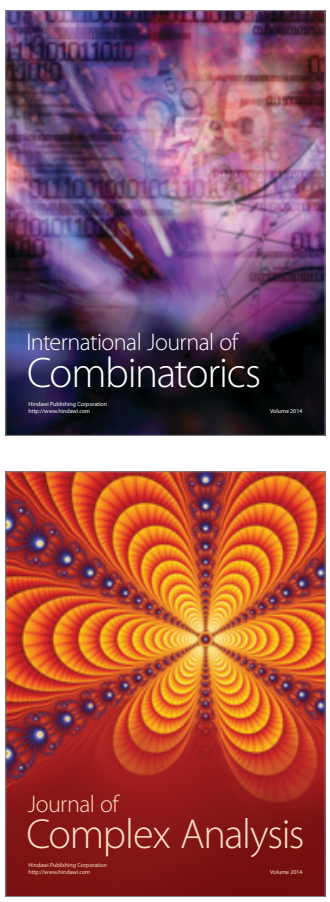

International Journal of

Mathematics and

Mathematical

Sciences
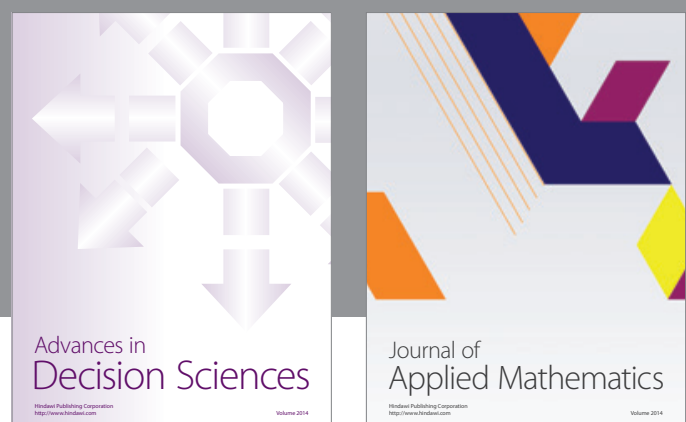

Journal of

Applied Mathematics
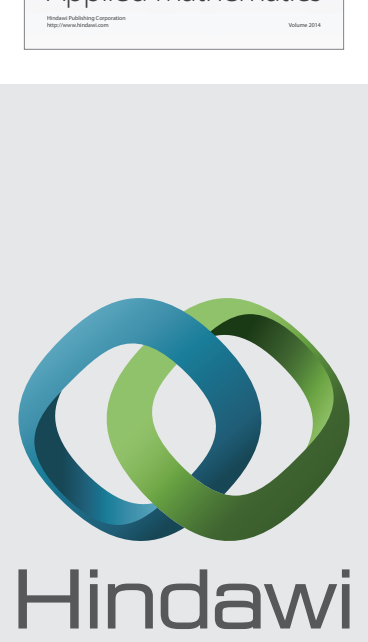

Submit your manuscripts at http://www.hindawi.com
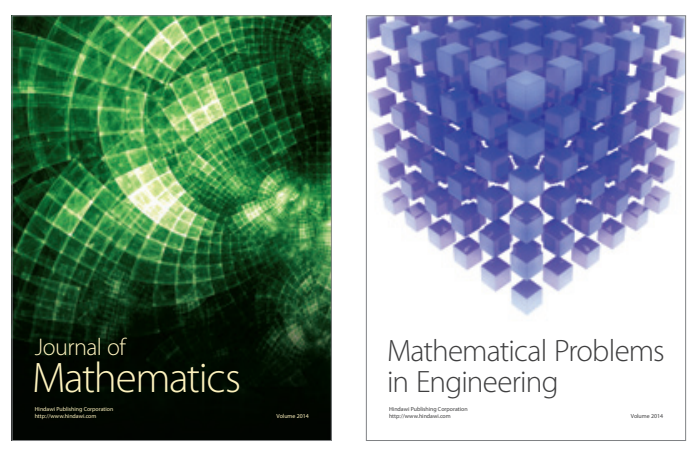

Mathematical Problems in Engineering
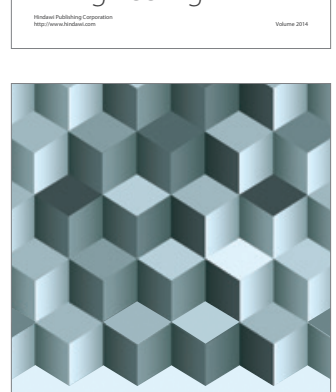

Journal of

Function Spaces
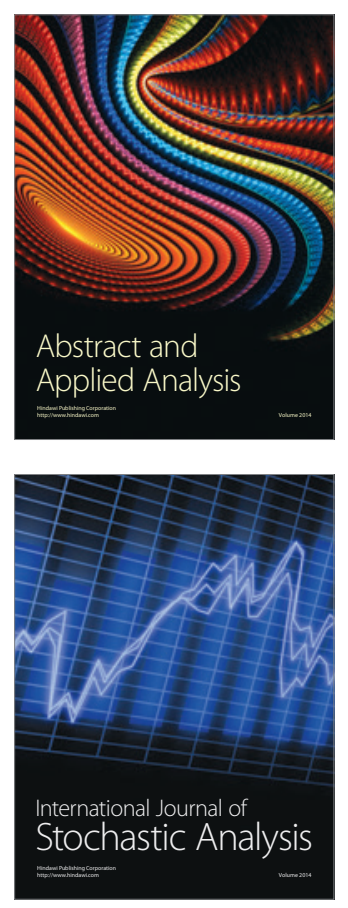

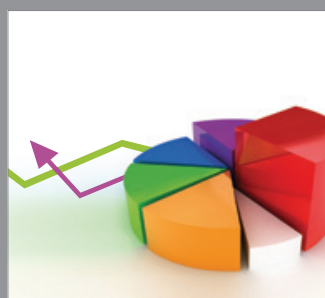

ournal of

Probability and Statistics

Promensencen
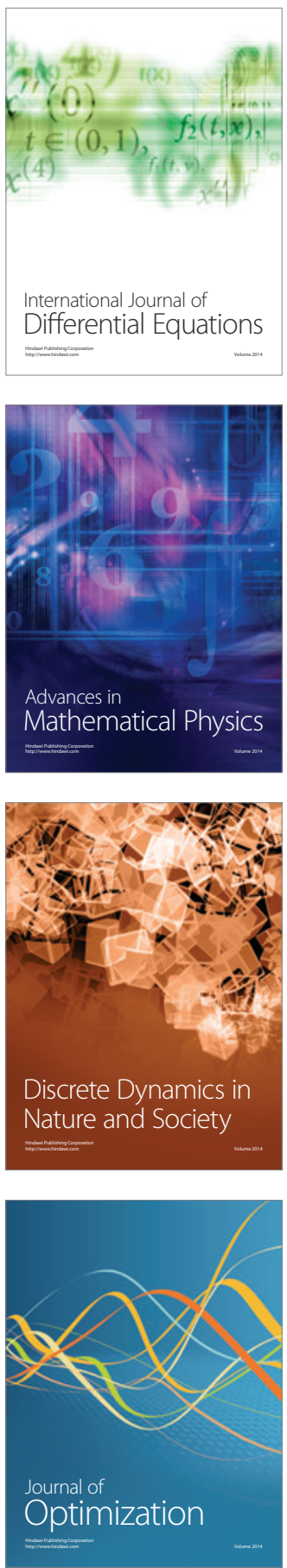